\title{
EVALUACIÓN DE DIFERENTES CONDICIONES DE PROCESO EN LA FORTIFICACIÓN DE MANGO (Tommy Atkins) CON CALCIO MEDIANTE IMPREGNACIÓN A VACÍO
}

\section{EVALUATION PROCESS IN DIFFERENT CONDITIONS OF MANGO FORTIFICATION (Tommy Atkins) WITH CALCIUM BY VACUUM IMPREGNATION}

\author{
Sandra Liliana Ostos A. (1), Amanda Consuelo Díaz M. (2), Héctor Suarez M. (2) \\ (1) Casa Luker S.A. Bogotá, Colombia. \\ (2) Instituto de Ciencia y Tecnología de Alimentos-ICTA. \\ Universidad Nacional de Colombia, Colombia
}

\begin{abstract}
Vacuum impregnation is an effective technology for the incorporation of physiologically active compounds in a food porous matrix within the framework of minimally processed, providing new product functionality to meet the needs of improving the nutritional quality of micronutrient-deficient populations. Fresh mango cylinders were vacuum impregnated with a solution of calcium lactate $6 \%$ and $1 \%$ calcium chloride, the process conditions (pressure and vacuum time) and sample size were defined by studying the interactions of which significantly affected parameters $X$ and ce impregnation. The technique allowed the incorporation of $37.6 \%$ (recommended daily intake) RDA of calcium in $200 \mathrm{~g}$ of fresh mango, variety Tommy Atkins, without affecting the sensory characteristics of the final product. The determination of calcium was performed by atomic absorption spectrophotometry. Process generated native fluid losses, expansion of fruit and low levels of $X$ and $\varepsilon e$ by the presence of calcium ions in the soaked mango.
\end{abstract}

Key words: Fortified foods, minerals, fruits, functional foods, mangoes.

Este trabajo fue recibido el 29 de Noviembre de 2011 y aceptado para ser publicado el 1 de Marzo de 2012.

\section{INTRODUCCIÓN}

La impregnación al vacío (IV) es un método reciente de procesamiento mínimo que aprovecha la micro estructura porosa de las matrices de los alimentos, para reemplazar el gas ocluido en su estructura celular por solutos contenidos en un líquido de impregnación mediante acción capilar y gradientes de presión impuestas en el sistema. Involucra un intercambio de materia promovido por la acción de un mecanismo hidrodinámico (MHD) entre un sistema sólido-líquido, acoplado a un fenómeno de deformación-relajación (FDR) en la matriz sólida del alimento. Este proceso está influenciado por la porosidad, propiedades mecánicas, tamaño y forma del alimento, viscosidad de la solución de impregnación o solución externa, propiedades físicas y químicas de los compuestos fisiológicamente activos, presiones y tiempos de vacío $(1,2)$.

En el primer paso del proceso, el alimento inmerso en la solución externa es expuesto a una presión negativa, aplicada por un tiempo suficiente hasta asegurar la remoción del aire atrapado en el espacio poroso del alimento y parte de la solución nativa (paso de vacío). Cuando es restaurada la presión atmosférica, genera una presión diferencial positiva que favorece la penetración de la solución externa dentro de los espacios libres de la estructura del alimento hasta alcanzar el equilibrio entre la presión interna y externa (3).

Como la técnica no involucra tratamientos térmicos, preserva el color, sabor natural, aroma y componentes nutritivos termosensibles. Este método ha sido ampliamente utilizado como pre tratamiento en procesos de secado, osmodeshidratación, congelación y en la 
prevención de pardeamiento oxidativo en frutas por remoción de oxígeno desde los poros (4). La técnica de impregnación al vacío tiene la capacidad de modificar la formulación de los alimentos y desarrollar ingredientes funcionales mediante la incorporación de compuestos fisiológicamente activos (CFA) como vitaminas, minerales, antioxidantes, enzimas, depresores de aw, reguladores de $\mathrm{pH}$, antimicrobianos, fibra dietaria, probióticos, prebióticos y simbióticos, mejorando las características sensoriales y nutricionales de los alimentos e incrementando la vida útil $(5,7$ - 11).

La tendencia del mercado es consumir alimentos saludables que ofrezcan bienestar al consumidor porque además de componentes nutritivos, contiene componentes que favorecen la salud, la capacidad física y el estado mental $(12,13)$. El enriquecimiento o fortificación de frutas con componentes fisiológicamente activos (CFA), como el calcio, podría jugar un papel importante en el bienestar de la población de los países pobres del mundo, porque contribuiría a disminuir la deficiencia del $85,8 \%$ en la ingesta de calcio (14).

El objetivo de esta investigación fue evaluar el enriquecimiento con calcio del mango a través de impregnación a vacío por medio de análisis fisicoquímico, reológico y sensorial.

\section{MATERIALES Y MÉTODOS}

La investigación fue realizada en tres etapas, la primera analizó las variables de proceso (presión y tiempo de vacío) y el tamaño de la muestra respecto a los parámetros de impregnación al vacío para definir las mejores condiciones (X, fracción volumétrica de la muestra impregnada por el líquido externo; g, deformación volumétrica de la muestra; $\varepsilon e$ porosidad efectiva de la muestra) según ecuación 1. En conclusión la fracción volumétrica de la muestra llenada con la solución externa (X) es una función de la porosidad efectiva del alimento (عe), velocidad de compresión aparente (r), deformación relativa de la muestra al final del paso 1 (g1) y deformación relativa de la muestra observada después del paso $2(\mathrm{~g})$, siendo esta última irreversible (1), donde la expresión de este modelo sería:

$$
\mathrm{r}(\mathrm{X}-\gamma)=\left(\varepsilon_{\mathrm{e}}+\gamma\right)(1-(1 / \mathrm{r}))-\gamma_{1} \quad \text { Ecuación } 1
$$

Por tanto la porosidad efectiva se calcula como:

$$
\varepsilon \mathrm{e}=\frac{(X-\gamma) r+\gamma_{1}}{r-1} \quad \text { Ecuación } 2
$$

La porosidad efectiva (ce) es expresada a priori como el porcentaje de volumen de muestra inicialmente ocupado por el gas pero se define más precisamente como la fracción de volumen de muestra disponible para un mecanismo HDM, este parámetro se calcula por la pendiente de la función lineal dada por la relación de $\mathrm{X}$ vs $1-1 / \mathrm{r}(1,15)$.

Los parámetros g1 y g están definidos como:

$$
\begin{aligned}
& \gamma_{1}:\left(\Delta \mathrm{V}_{1} / \mathrm{V}_{\mathrm{o}}\right) \\
& \gamma:\left(\Delta \mathrm{V} / \mathrm{V}_{\mathrm{o}}\right)
\end{aligned}
$$

Donde $\Delta \mathrm{V}_{1}$ y $\mathrm{V}_{\mathrm{o}}$ son el volumen de deformación de la muestra al final del paso de vacío y al final del paso de presión atmosférica, respectivamente, y Vo, es el volumen de la muestra inicial.

En este sentido, si $(X-\gamma) r>\gamma_{1}$, la contribución de $\gamma_{1}$ para la evaluación del X no es significativa. Por tanto, la fracción volumétrica del alimento poroso impregnado durante una impregnación al vacío puede ser modelado como:

$$
\mathrm{X}=\varepsilon_{\mathrm{e}}(1-1 / \mathrm{r})+\gamma \quad \text { Ecuación } 3
$$

En la segunda etapa fue analizada la capacidad de fortificación del mango con diferentes sales de calcio (cloruro de calcio, lactato de calcio, gluconato de calcio, fumarato de calcio) para obtener un aporte del $20 \%$ de la ingesta diaria recomendada (IDR) en $200 \mathrm{~g}$ de mango fresco. En la tercera etapa se aplicó la técnica de impregnación al vacío y cuantificación del calcio incorporado en el mango.

\section{Materia prima}

Se seleccionaron mangos Tommy Atkins entre 10-12oBrix con tamaños entre 400 g y 500 g, corteza firme, sin evidencia de daño mecánico e infestación. El estado de madurez de la fruta fue seleccionado en base al criterio industrial de compra de materia prima y por la resistencia de la fruta a la manipulación del proceso de impregnación a vacío. Las muestras fueron acondicionadas, lavadas, peladas y cortadas utilizando un molde cilíndrico de acero inoxidable.

Como líquido de impregnación fue utilizado inicialmente una solución isotónica de sacarosa $16^{\circ}$ Brix con aw similar a la del mango (ver tabla 1 ) y como medio de fortificación fue seleccionada la mezcla de sales de calcio (lactato de calcio pentahidratado al $6 \%$ y cloruro de calcio dihidratado al 1\%) en solución de sacarosa $16^{\circ}$ Brix.

\section{Preparación de la muestra}

Las muestras cortadas en cilindros $\left(\mathrm{G}_{1}\right.$ : diámetro 20 $\pm 2 \mathrm{~mm}$ y altura $10 \pm 2 \mathrm{~mm} ; \mathrm{G}_{2}$ : diámetro $20 \pm 2 \mathrm{~mm}$ y altura $20 \pm 2 \mathrm{~mm}$ ) fueron sumergidas solución isotónica de sacarosa y colocadas dentro de la cámara de vacío, 
fueron utilizadas dos presiones de vacío $\left(\mathrm{P}_{1}\right.$ y $\left.\mathrm{P}_{2}\right)$ y dos tiempos de vacío $\left(\mathrm{t}_{1} \mathrm{y}_{2}\right.$ ) (tabla 4$)$, posteriormente restablecida la presión atmosférica a $646 \mathrm{~mm} \mathrm{Hg}$. Las muestras se mantuvieron sumergidas durante 5 minutos. A cada muestra impregnada fueron determinados los parámetros de impregnación. Con las mejores condiciones de tiempo, presión y tamaño fue realizada la incorporación del calcio y calculados nuevamente los parámetros de impregnación.

\section{Caracterización fisicoquímica}

A la fruta fresca se le determinó la acidez titulable, $\mathrm{pH}$, sólidos solubles, humedad, actividad de agua, densidad real y aparente, porosidad y contenido de calcio. A la muestra impregnada se le realizaron análisis de color, textura, sensorial, contenido de calcio. Todos los análisis se realizaron por triplicado.

\section{Acidez titulable y pH}

La acidez titulable fue determinada usando el método AOAC 942.15A, por titulación con la solución de hidróxido de sodio $0,1 \mathrm{~N}$ y expresada como porcentaje (\%) de ácido cítrico por $100 \mathrm{ml}$ de producto. La valoración potenciométrica fue realizada por el método AOAC 981.12, con un pH-metro ORION modelo 420, previamente calibrado con soluciones buffer de $\mathrm{pH} 4$ y 7 .

\section{Sólidos solubles}

Para la medición de sólidos solubles fue empleado el método refractométrico AOAC 932.12 y realizada la lectura en un refractómetro digital marca HANNA HI96801 (0-32\%).

\section{Humedad y actividad de agua}

La medición del contenido de humedad se realizó por el método gravimétrico según la AOAC 931.04, en horno a $100{ }^{\circ} \mathrm{C}$ hasta obtener peso constante.

La actividad de agua $\left(\mathrm{a}_{\mathrm{w}}\right)$ fue realizada por el método AOAC 978.18, utilizando un higrómetro de punto de rocío marca AQUALAB DECAGÓN 3TE.

\section{Densidad real $\left(p_{r}\right)$ y aparente $\left(p_{\text {apm }}\right)$ del mango}

La medición de la densidad aparente $\left(\mathbf{p}_{\text {apm }}\right)$ del mango fue por desplazamiento de volumen empleando una probeta con agua a $20^{\circ} \mathrm{C}$. La densidad real del mango $\left(\mathrm{p}_{\mathrm{r}}\right)$ fue determinada a partir del contenido de humedad $\left(\mathrm{x}_{\mathrm{w}}\right)$, donde fue considerado que la muestra está compuesta únicamente por carbohidratos y agua.

\section{Porosidad real del mango $(\varepsilon)$}

Para determinar la porosidad real del mango $(\varepsilon)$ fue utilizada la relación entre densidad real de la fruta $\left(\mathrm{p}_{\mathrm{r}}\right)$ y densidad aparente $\left(\mathrm{p}_{\mathrm{a}}\right)(15,16)$.

\section{Determinación de calcio}

El contenido de calcio fue realizado según modificación del método AOAC 965.09, descrito a continuación:

Fueron pesados en crisol aproximadamente $12 \mathrm{~g}$ de mango fresco y mango impregnado, la muestra fue precalcinada y posteriormente calcinada en mufla a $550^{\circ} \mathrm{C}$ durante 4 horas. La digestión de la muestra fue realizada con $3 \mathrm{~mL}$ de ácido nítrico concentrado y $1 \mathrm{~mL}$ de ácido clorhídrico concentrado calentando suavemente. La solución fue filtrada con papel $3 \mathrm{HW}$ y completado el volumen a $100 \mathrm{ml}$ en balón aforado.

Para el mango impregnado, de esta solución fue utilizado $1 \mathrm{~mL}$ y completado a $50 \mathrm{~mL}$ con agua desionizada. Para el mango fresco fue utilizada una alícuota de $5 \mathrm{~mL}$ y completada a $25 \mathrm{~mL}$ con agua desionizada. Antes de completar el volumen fue adicionado $2,5 \mathrm{~mL}$ de cloruro de lantánido $\left(\mathrm{LaCl}_{3}\right)$ al $1 \%$ p/p y $2,5 \mathrm{ml}$ de cloruro de potasio $(\mathrm{KCl}) 20000 \mathrm{ppm}$ para eliminar interferencia de otros minerales. La curva de calibración fue construida entre 0,25 y $3,50 \mathrm{ppm}$ de carbonato de calcio $\left(\mathrm{CaCO}_{3}\right)$.

La cuantificación del calcio fue realizada utilizando un espectrómetro de absorción atómica marca Varian AA240 con quemador de aire, óxido nitroso y acetileno y lámpara de cátodo hueco Varian Calcium HC. La longitud de onda utilizada para la lectura fue de 422,7 nm.

\section{Análisis de color}

El color fue medido utilizando un colorímetro SP64, Marca X-RITE, empleando el iluminante $\mathrm{D}_{65} \mathrm{y}$ el observador de $10^{\circ}$ como referencia. A partir del espectro de reflectancia de las muestras fueron determinadas las coordenadas de color CIE-LAB, L* (luminosidad), $\mathrm{a}^{*}$ (cromaticidad de verde a rojo) y $\mathrm{b}^{*}$ (cromaticidad de azul a amarillo).

\section{Análisis de textura}

La textura se determinó midiendo la fuerza de ruptura utilizando el analizador de textura TA:XT2, Stable Micro Systems, empleando una sonda de $4 \mathrm{~mm}$ de diámetro, velocidad de carga de 2 o $4 \mathrm{~mm} / \mathrm{s}$ y distancia de penetración 10 a $5 \mathrm{~mm}$. Los valores fueron calculados según la curva de fuerza máxima (F) Vs distancia (D) (17).

\section{Evaluación sensorial}

Fueron utilizados 8 jueces entrenados que cataron de manera simultánea y aleatoria, muestras de mango impregnado con diferentes concentraciones de sales y muestras de mango fresco como patrón de referencia. Los atributos evaluados fueron: aroma, color, sabor, 
acidez y dureza; empleando respuestas numéricas en una escala de medición ordinal. Inicialmente fue analizado el acuerdo entre jueces a través del coeficiente de concordancia de Kendall y luego aplicado el test de Wilcoxon para encontrar diferencias significativas entre las muestras impregnadas y el patrón de referencia (18).

\section{Diseño experimental y análisis estadístico}

Para evaluar la influencia del tamaño de la muestra $(\mathrm{G})$, la presión de vacío $(\mathrm{P})$ y tiempo de vacío (t) fue utilizado un diseño multifactorial de 3 factores $(\mathrm{G}, \mathrm{P}, \mathrm{t})$ con dos niveles cada uno $\left(\mathrm{G}_{1}, \mathrm{G}_{2}, \mathrm{P}_{1}, \mathrm{P}_{2}, \mathrm{t}_{1}, \mathrm{t}_{2}\right)$, de efectos fijos completamente aleatorizados, con 10 repeticiones en cada celda. El nivel de significancia empleado en el tratamiento estadístico de datos fue de 0,05 , los niveles se discriminaron utilizando el Test de Tukey. Los resultados fueron analizados en Statgraphics STSC Inc., Rockville, MD, USA, 1993.

\section{RESULTADOS Y DISCUSIÓN Análisis fisicoquímico de las muestras}

La tabla 1 presenta los valores promedio y desviación estándar de los parámetros del análisis fisicoquími- co del mango fresco y los valores reportados por otros autores $(16,19,20)$.

La porosidad real fue mayor a la porosidad efectiva porque todo el volumen libre de la fruta no es completamente llenado por la disolución debido a los efectos de la capilaridad y modificaciones estructurales ocasionados por los cambios de presión del sistema, coincidiendo con lo señalado por otros autores (19), además es necesario considerar el efecto de la viscosidad de la disolución que determina la entrada del líquido en los poros de la matriz, reportado igualmente en otros trabajos $(3,21)$.

La relación entre porosidad efectiva $\left(\varepsilon_{\mathrm{e}}\right)$ y porosidad real del mango $(\varepsilon), \varepsilon_{\mathrm{e}} / \varepsilon$, varía entre autores, algunos resultados indican valores entre 0,59-0,76 (20), sin embargo otros autores reportan valores que oscilan entre 0,105 y $3,00(18)$.

\section{Proceso de impregnación a vacío}

La tabla 2 presenta los valores promedio y la desviación estándar de los parámetros de impregnación correspondiente a cada tamaño de muestra de mango $(\mathrm{G})$; a las variables del proceso presión $(\mathrm{P})$ y tiempo $(\mathrm{t})$ de vacío con solución isotónica de sacarosa de $16^{\circ}$ Brix

TABLA 1

\section{Caracterización fisicoquímica del mango fresco}

\section{Característica}

Actividad de agua

Humedad (\%)

$\mathrm{pH}$

Acidez ( $\mathrm{g}$ de ácido

cítrico/100 g muestra)

Sólidos solubles

( ${ }^{\circ}$ Brix)

Índice de madurez

Densidad aparente

del mango $\left(\mathrm{kg} / \mathrm{m}^{3}\right)$

Densidad real del

mango $\left(\mathrm{kg} / \mathrm{m}^{3}\right)$

Porosidad real del

mango $(\varepsilon)$

Porosidad efectiva

del mango $\left(\varepsilon_{\mathrm{e}}\right)$

Relación $\varepsilon$ e / $\varepsilon$

\section{Resultados}

Cortés et al., 2007 (Var. Tommy Atkins)
Mujica-Paz et al., 2003 (Var. Manila)

Salvatori et al., 1998 (Var. Tommy Atkins)

$0,983+0,002$

$83,6+0,5$

3,2

$3,46+0,01$

$1,124+0,05$

$1,4+0,2$

$11,3+0,7$

$10,0+0,1$

$10,50+1,5 \quad 7,3+0,9$

$961,2+0,1$

$987,3+0,1$

$1054,5+1,6$

$1057,8+1,9$

$0,088+0,001$

$0,066+0,001$

$0,064+0,006$

$0,073+0,008$

0,727
$0,988+0,001$

$84,1+0,7$

3,0

$1,37+0,01$

$16,1+0,3$

11,7

$0,918+0,01$

$1082+0,02$

$0,152+0,001$

0,016

0,697
$0,9895+0,0007$

$0,7868+0,0007$

4,29

$0,408+0,009$

$17,8+0,5$

$43,69+0,16$

$1022+0,005$

$1130+0,2$
1,107

0,596 
como líquido de impregnación.

En general, los valores de porosidad efectiva $\left(\varepsilon_{\mathrm{e}}\right)$ obtenidos con la disolución isotónica muestran disponibilidad de los espacios intercelulares para la incorporación de la disolución. La mayor fracción volumétrica fue obtenida a $138 \mathrm{mmHg}$ de presión de vacío y el mango acondicionado en cilindros de h: $10 \mathrm{~mm}$ y D: $20 \mathrm{~mm}$, porque con esta relación es mayor la extracción de aire y el área superficial del fruto. A diferencia del tiempo de vacío, cuyo efecto fue inversamente proporcional respecto a la fracción volumétrica (X), siendo el mejor tiempo de vacío: $5 \mathrm{~min}$. En este sentido, las condiciones de proceso definidas para el estudio son: presión de vacío (138 mmHg), tiempo de vacío (5 min) y tamaño de muestra (Cilindros, h: $10 \mathrm{~mm}$ y D: $20 \mathrm{~mm}$ ).Al evaluar el modelo factorial completo (efectos principales y la interacción total; G, P, t), se puede concluir que existen diferencias significativas entre las medias de $\mathrm{X}$ y de $\varepsilon$ e.
Las interacciones entre $\mathrm{G}$ y P, Py t, afecta las medias de $\mathrm{X}$ y $\varepsilon_{\mathrm{e}}$, mientras que la interacción entre $\mathrm{G}$ y t no afectan significativamente las medias de $\varepsilon e$, situación que se observa con la mayor presión de vacío $(138 \mathrm{mmHg})$. De igual manera, la interacción entre $\mathrm{G}$ y t no afectan significativamente las medias de $\mathrm{X}$, situación que se observa con la menor presión de vacío $(138 \mathrm{mmHg})$ y mayor tamaño de muestra (Cilindros, D (20 \pm 2$) \mathrm{mm}$; h: (20 \pm 2$) \mathrm{mm}$.

En la tabla 3 se presentan los valores experimentales obtenidos de fracción volumétrica $(\mathrm{X})$ y la porosidad efectiva $\left(\varepsilon_{\mathrm{e}}\right)$ y se muestran los resultados de otros autores (16). Los mejores resultados de $\mathrm{X}$ y $\varepsilon_{\mathrm{e}}$ se obtuvieron utilizando $138 \mathrm{mmHg}$ de presión de vacío; 5 min de tiempo de vacío; $646 \mathrm{mmHg}$ y $5 \mathrm{~min}$ a presión atmosférica; en cilindros de $20 \mathrm{~mm} \pm 2 \mathrm{~mm}$ de diámetro y $10 \mathrm{~mm} \pm 2$ mm de altura; solución de impregnación: Sacarosa 160 Brix; relación fruta/LI: 4/100.

\section{TABLA 2}

\section{Parámetros de impregnación, fracción volumétrica (X) y porosidad efectiva (ce), a diferentes condiciones de proceso (presión y tiempo de vacío) y tamaño de muestra.}

\begin{tabular}{|c|c|c|c|c|c|c|c|c|}
\hline \multirow{3}{*}{$\begin{array}{c}\text { Tamaño } \\
\text { muestra } \\
(\mathbf{G})\end{array}$} & \multicolumn{6}{|c|}{ Condiciones de proceso: presión $(P)$ y tiempo $(t)$ de vacío } & \multirow{2}{*}{\multicolumn{2}{|c|}{$\begin{array}{c}\left(\mathbf{P}_{2}: 138 \mathrm{mmHg}\right. \\
\left.\mathrm{t}_{2}: 10 \mathrm{~min}\right)\end{array}$}} \\
\hline & \multicolumn{2}{|c|}{$\begin{array}{l}\left(\mathbf{P}_{1}: 392 \mathrm{mmHg}\right. \\
\left.\mathrm{t}_{1}: 5 \mathrm{~min}\right)\end{array}$} & \multicolumn{2}{|c|}{$\begin{array}{c}\left(\mathbf{P}_{1}: 392 \mathrm{mmHg}\right. \\
\left.\mathrm{t}_{2}: 10 \mathrm{~min}\right)\end{array}$} & \multicolumn{2}{|c|}{$\begin{array}{c}(\mathrm{P} 2: 138 \mathrm{mmHg}, \\
\left.\mathrm{t}_{1}: 5 \mathrm{~min}\right)\end{array}$} & & \\
\hline & $\mathbf{X}$ & $\varepsilon_{\mathrm{e}}$ & $\mathbf{X}$ & $\varepsilon_{\mathrm{e}}$ & $\mathbf{X}$ & $\varepsilon_{\mathrm{e}}$ & $\mathbf{X}$ & $\varepsilon_{\mathrm{e}}$ \\
\hline $\begin{array}{l}\text { Cilindros } \\
\mathrm{D}(20 \pm 2) \mathrm{mm} \\
\mathrm{h}(10 \pm 2) \mathrm{mm}\end{array}$ & $5,489 \pm 0,825^{\mathrm{a}}$ & $5,788 \pm 0,526^{a}$ & $3,685 \pm 0,793^{c}$ & $6,900 \pm 0,690^{c}$ & $7,524 \pm 0,599^{e}$ & $7,963 \pm 0,539^{e}$ & $6,890 \pm 0,980^{g}$ & $7,625 \pm 0,854^{e}$ \\
\hline $\begin{array}{l}\text { Cilindros } \\
\mathrm{D}(20 \pm 2) \mathrm{mm} \\
\mathrm{h}(20 \pm 2) \mathrm{mm}\end{array}$ & $2,312 \pm 0,672^{b}$ & $2,639 \pm 0,620^{\mathrm{b}}$ & $2,380 \pm 0,574^{b}$ & $4,051 \pm 0,595^{\mathrm{d}}$ & $6,170 \pm 0,385^{f}$ & $6,362 \pm 0,621^{\mathrm{f}}$ & $5,137 \pm 0,876^{\mathrm{h}}$ & $5,868 \pm 0,853^{\mathfrak{f}}$ \\
\hline
\end{tabular}

TABLA 3

Comparación de los parámetros de impregnación $\left(X, \varepsilon_{\mathrm{e}}\right)$ respecto a otro autor

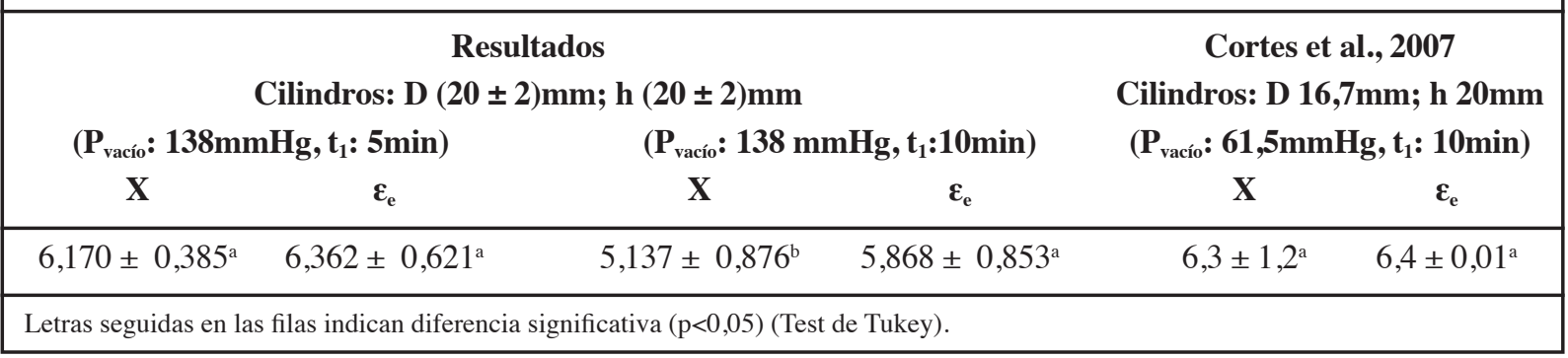




\section{Selección de las sales de calcio}

La selección de las sales de calcio que permite incorporar $20 \%$ de la ingesta diaria recomendada (IDR) en 200 $\mathrm{g}$ de fruta fresca, fue obtenida calculando teóricamente el contenido de calcio en la disolución de impregnación, de acuerdo con el balance de materia propuesto por otros autores (16) y determinando la concentración de calcio en la matriz del mango en diferentes concentraciones de sales por espectrofotometría de masas (tabla 4). Los valores de IDR obtenidos del lactato de calcio $6 \%$ y $7 \%$ son similares porque por encima del $6 \%$ se presenta saturación de la sal en el líquido de impregnación. Las mezclas de sales aportaron valores de IDR superiores al $20 \%$ por efecto de la salida de líquido nativo presente en el mango.

\section{Análisis sensorial}

El análisis sensorial con panelistas entrenados fue el último criterio para definir la composición de la mezcla de sales. En la tabla 5 se presentan los resultados de la evaluación de los atributos sensoriales del mango fresco e impregnado con sales de calcio. Para esta evaluación fue seleccionada la mezcla de lactato de calcio $6 \%$, cloruro de calcio $1 \%$ porque no existe diferencia significativa $(p>0,05)$ y ofrecía el mayor aporte de calcio.

Previo a la comparación sensorial de sales, fue aplicado el test de Kendall para analizar la concordancia entre los resultados de los jueces, como el valor $\mathrm{p}$ fue 0,813 , existe acuerdo, es decir, los panelista entrenados son confiables para la prueba.

El test de Wilcoxon indica que el mango impregnado con las mezclas 1 y 2 no presenta diferencia significativa en los atributos evaluados respecto al mango fresco. El mango impregnado con la mezcla 3 presenta diferencias significativas $(\mathrm{p}<0,05)$ en todas las variables excepto en la acidez.

Los incrementos en la dureza de la fruta asociados a la mayor concentración de calcio podrían estar relacionados con la formación de geles, debido a la desesterificación de las pectinas que refuerza la estructura de la fruta, coincidiendo con otros autores (9). El cambio en el color es explicado por la presencia de líquido de impregnación en los espacios intercelulares del mango.

\section{Impregnación a vacío con sales de calcio}

En la tabla 6 se muestran los valores promedio y desviación estándar de todos los parámetros de impregnación obtenidos a partir de la disolución de impregnación con sacarosa y sales de calcio $\mathrm{Ca}^{+2}$. El proceso de impregnación con la disolución de sales, produce una reducción en los valores de $\mathrm{X}$ y $\varepsilon_{\mathrm{e}}$ respecto a la disolución isotónica, indicando una mayor contracción del volumen de poros durante el proceso. Este resultado es atribuible principalmente a las interacciones de las pectinas del

\section{TABLA 4}

Concentración de calcio en mango impregnado con diferentes mezclas de sales de calcio.

\begin{tabular}{|ccc|}
\hline Muestra & $\begin{array}{c}\text { mgCa+2 } \mathbf{e n ~ 2 0 0 g} \\
\text { de mango fresco }\end{array}$ & $\begin{array}{c}\text { \% IDR Ingesta } \\
\text { Diaria Recomendada }\end{array}$ \\
\hline $\begin{array}{c}\text { Mango fresco } \\
\text { Mango impregnado }\end{array}$ & $16,88 \pm 0,14$ & $1,69 \pm 0,01$ \\
(lactato calcio 6\%, cloruro de calcio 1\%) & $317,18 \pm 1,82^{\mathrm{a}}$ & $31,72 \pm 0,18 \mathrm{a}$ \\
Mango fresco & $18,72 \pm 0,27$ & $1,87 \pm 0,03$ \\
Mango impregnado & $245,20 \pm 1,21 \mathrm{~b}$ & $24,52 \pm 0,12 \mathrm{~b}$ \\
Mango fresco & $17,65 \pm 0,32$ & $1,77 \pm 0,03$ \\
Mango impregnado & & $18,50 \pm 0,24 \mathrm{c}$ \\
(lactato calcio 7\%) & $185,03 \pm 2,41 \mathrm{c}$ & $1,74 \pm 0,04$ \\
Mango fresco & $17,42 \pm 0,43$ & $18,46 \pm 0,20 \mathrm{c}$ \\
\hline $\begin{array}{c}\text { Mango impregnado } \\
\text { (lactato calcio 6\%) }\end{array}$ & $184,55 \pm 1,97 \mathrm{c}$ & \\
\hline Letras seguidas en filas indican diferencia significativa (p<0,05). (Test de Tukey). & \\
\hline
\end{tabular}


mango con los iones $\mathrm{Ca}^{+2}$ presentes en la disolución, las cuales inducen a un sistema gelificado en el interior de la estructura incrementando la caída de presión del líquido a la entrada del poro, igualmente reportado por otros autores (16).

La presencia de valores $\mathrm{X}_{1}$ negativos pueden ser explicados por la pérdida de líquidos nativos desde los espacios intercelulares producidos durante la expansión del gas ocluido. La pérdida de líquidos nativos se incrementa con la disolución de fortificación por el intercambio de materia que ocurre durante la impregnación.

Los valores positivos de deformación $\gamma_{1}$ y $\gamma$ indican que hubo expansión de la fruta durante el proceso, por lo tanto el poro se incrementó con respecto al valor inicial, como consecuencia de la expansión inicial del gas. Este fenómeno se presenta con solución isotónica de sacarosa y con sales de calcio.

Las variables de impregnación (X, $\left.\mathrm{X}_{1}, \gamma_{1}, \gamma, \varepsilon_{\mathrm{e}}\right)$ presentaron diferencias estadísticamente significativas $(\mathrm{p}<0.05)$ entre la solución isotónica y la solución enriquecida de calcio.

\section{Color, dureza y concentración de calcio}

Las curvas de fuerza/deformación muestran que el mango impregnado es menos elástico que el mango fresco por el estrés mecánico al que ha sido sometida la fruta y por efecto del gel formado al reaccionar iones calcio con los grupos carboxílico del ácido pectínico (8) (tabla 7). Estos datos presentan correlación con los resultados sensoriales del mango impregnado (tabla 5) y

TABLA 5

Atributos sensoriales del mango fresco e impregnado con sales de calcio

\begin{tabular}{|lcccc|}
\hline $\begin{array}{l}\text { Atributos } \\
\text { Sensoriales }\end{array}$ & $\begin{array}{c}\text { Mango } \\
\text { fresco }\end{array}$ & $\begin{array}{c}\text { Mezcla 1: } \\
\text { lactato de calcio 6\% } \\
\text { Cloruro de calcio 0,5\% }\end{array}$ & $\begin{array}{c}\text { Mango Impregnado } \\
\text { Mezcla 2: } \\
\text { lactato de calcio 6\% } \\
\text { Cloruro de calcio 1\% }\end{array}$ & $\begin{array}{c}\text { Mezcla 3: } \\
\text { lactato de calcio 7\% } \\
\text { Cloruro de calcio 1\% }\end{array}$ \\
\hline Aroma & $5,3 \mathrm{a}$ & $5,0 \mathrm{a}$ & $5,0 \mathrm{a}$ & $4,5 \mathrm{~b}$ \\
Color & $5,5 \mathrm{~b}$ & $4,5 \mathrm{~b}$ & $5,0 \mathrm{~b}$ & $4,3 \mathrm{c}$ \\
Sabor & $4,5 \mathrm{c}$ & $4,0 \mathrm{c}$ & $4,5 \mathrm{c}$ & $3,8 \mathrm{~d}$ \\
Acidez & $4,0 \mathrm{e}$ & $3,8 \mathrm{e}$ & $3,8 \mathrm{e}$ & $3,8 \mathrm{e}$ \\
Dureza & $4,0 \mathrm{f}$ & $5,0 \mathrm{f}$ & $5,0 \mathrm{f}$ & $6,0 \mathrm{~h}$ \\
\hline Letras seguidas en filas y columnas indican diferencia significativa $(\mathrm{p}<0,05)($ Test de Wilcoxon). & \\
\hline
\end{tabular}

\section{TABLA 6}

Parámetros de impregnación $\left(\mathbf{X}, \mathbf{X}_{1}, \gamma, \gamma_{1}\right)$ con disolución isotónica de sacarosa y mezcla de sales.

\begin{tabular}{|c|c|c|c|c|c|c|c|c|c|c|}
\hline \multirow{3}{*}{$\begin{array}{l}\text { Condiciones } \\
\text { Tamaño } \\
\text { muestra (G) }\end{array}$} & \multicolumn{10}{|c|}{ (P1: 138mmHg, t1: 5min) } \\
\hline & \multicolumn{5}{|c|}{ Solución Isotónica } & \multicolumn{5}{|c|}{ Lactato de calcio $6 \%$ - Cloruro de calcio $1 \%$} \\
\hline & $\gamma$ & $\gamma_{1}$ & $\mathrm{X} 1$ & $\mathbf{X}$ & $\varepsilon_{\mathrm{e}}$ & $\gamma$ & $\gamma_{1}$ & $\mathbf{X}_{1}$ & $\mathbf{X}$ & $\varepsilon_{\mathrm{e}}$ \\
\hline \multicolumn{11}{|l|}{ Cilindros } \\
\hline D $20 \pm 2 \mathrm{~mm}$ & 2,169 & 3,822 & (-) 8,430 & 7,524 & 7,963 & 4,096 & 8,990 & (-)12,631 & 3,659 & 4,630 \\
\hline h $10 \pm 2 \mathrm{~mm}$ & $\pm 0,740^{\mathrm{a}}$ & $\pm 0,895^{\mathrm{A}}$ & $\pm 0,883^{\text {aa }}$ & $\pm 0,599^{\mathrm{AA}}$ & $\pm 0,539^{\text {aaa }}$ & $\pm 0,887^{\mathrm{c}}$ & $\pm 0,950^{\mathrm{b}}$ & $\pm 0,964^{\mathrm{bb}}$ & $\pm 1,006^{\mathrm{cc}}$ & $\pm 0,982^{\mathrm{ccc}}$ \\
\hline \multicolumn{11}{|l|}{ Cilindros } \\
\hline D $20 \pm 2 \mathrm{~mm}$ & 1,243 & 3,391 & (-) 5,450 & 6,170 & 6,362 & 2,595 & 5,274 & (-) 6,635 & 3,570 & 4,237 \\
\hline h $10 \pm 2 \mathrm{~mm}$ & $\pm 0,389^{\mathrm{b}}$ & $\pm 0,641^{\mathrm{A}}$ & $\pm 0,771^{\mathrm{ab}}$ & $\pm 0,385^{\mathrm{BB}}$ & $\pm 0,621^{\mathrm{bbb}}$ & $\pm 0,640^{\mathrm{d}}$ & $\pm 0,777^{\mathrm{c}}$ & $\pm 0,380^{\mathrm{cc}}$ & $\pm 0,727^{\mathrm{cc}}$ & $\pm 0,263^{\mathrm{ccc}}$ \\
\hline
\end{tabular}


con lo reportado por otros autores $(5,6)$, donde utilizando manzanas impregnadas con calcio (mezcla de lactato y gluconato de calcio) y comparadas con la fruta fresca, presentan disminución en los valores de la fuerza de ruptura y fractura.

El color de la fruta impregnada respecto a la fruta fresca, presenta disminución en los parámetros de luminosidad $\left(\mathrm{L}^{*}\right)$, cromaticidad de verde a rojo $\left(\mathrm{a}^{*}\right)$ y cromaticidad de azul a amarillo (b*) (tabla 7), situación que es explicada por la presencia de líquido de impregnación en los espacios intercelulares del mango fortificado (6). Este resultado no presenta correlación con el análisis sensorial.

\section{Niveles de calcio obtenidos}

La tabla 8 , presenta los niveles de $\mathrm{Ca}^{+2}$ obtenidos para el mango fresco y para el mango impregnado con calcio. El mango enriquecido representa un 37,6\% de la IDR, concentración que demuestra la efectividad del proceso de impregnación a vacío como metodología de enriquecimiento.
Los estudios realizados hasta la fecha a nivel de laboratorio demuestran que la técnica de IV permite incorporar calcio en frutas hasta en un $62 \%$ de la IDR. Comparado con otros autores (15) con similares características del fruto, se obtuvieron en este estudio valores de IDR $14,4 \%$ por debajo, debido a las diferencias en las presiones de vacío.

\section{CONCLUSIONES}

Este estudio demostró que el proceso de impregnación a vacío permite incorporar hasta en un $37,6 \%$ de la IDR de $\mathrm{Ca}^{+2} / 200 \mathrm{~g}$ de mango sin afectar las características sensoriales del producto fortificado, siendo una metodología eficaz para desarrollar ingredientes funcionales.

Los parámetros de impregnación ( $\mathrm{X}$ y $\varepsilon_{\mathrm{e}}$ ) se afectaron significativamente por la interacción de las variables geometría de la muestra, tiempo y presión de vacío. Estos valores se disminuyen por la presencia de iones calcio en la fruta.

Hubo pérdidas de líquidos nativos (valores $\mathrm{X}_{1}$ ne-

TABLA 7

Características físicas del mango fresco e impregnado.

\begin{tabular}{|lcccc|}
\hline Muestra & $\begin{array}{c}\text { Textura } \\
\text { Fuerza de ruptura (kg/fuerza) }\end{array}$ & $\mathbf{L}^{*}$ & $\begin{array}{c}\text { Color } \\
\mathbf{a}^{*}\end{array}$ & $\mathbf{b}^{*}$ \\
\hline \multirow{2}{*}{ Mango fresco } & $0,741 \pm 0,139^{\mathrm{a}}$ & $67,48 \pm 4,14^{\mathrm{a}}$ & $7,51 \pm 2,55^{\mathrm{a}}$ & $54,22 \pm 3,93^{\mathrm{a}}$ \\
Mango impregnado & $0,576 \pm 0,079^{\mathrm{b}}$ & $51,21 \pm 4,06^{\mathrm{b}}$ & $3,90 \pm 1,54^{\mathrm{b}}$ & $40,86 \pm 3,96^{\mathrm{b}}$ \\
\hline Letras seguidas en columnas indican diferencia significativa $(\mathrm{p}<0,05)($ Test de Tukey). & & \\
\hline
\end{tabular}

\section{TABLA 8}

Comparación entre niveles de calcio obtenidos y otro autor.

\begin{tabular}{|c|c|c|c|c|}
\hline \multirow[b]{2}{*}{ Muestra } & \multicolumn{2}{|c|}{$\begin{array}{c}\text { Resultados en el laboratorio } \\
\text { Cilindros } \\
\text { D }(20 \pm 2) \mathrm{mm}-\mathrm{h}(10 \pm 2) \mathrm{mm} \\
\text { P }_{\text {vacio }}: 138 \mathrm{mmHg}, \mathrm{t} 1: 5 \mathrm{~min}\end{array}$} & \multicolumn{2}{|c|}{$\begin{array}{c}\text { Cortés et al., } 2007 \\
\text { Cilindros } \\
\mathrm{D}(\mathbf{2 0} \pm \mathbf{2}) \mathrm{mm}-\mathrm{h}(10 \pm 2) \mathrm{mm} \\
\mathbf{P}_{\text {vacío }}: \mathbf{6 1 , 5} \mathrm{mmHg}, \mathrm{t} 1: \mathbf{1 0 m i n}\end{array}$} \\
\hline & $\begin{array}{l}\mathrm{mg} \mathrm{Ca}+2 / 200 \mathrm{~g} \\
\text { Mango fresco }\end{array}$ & $\begin{array}{l}\text { Ingesta diaria } \\
\text { Recomendada }\end{array}$ & $\begin{array}{c}\mathrm{mg} \mathrm{Ca}+2 / 200 \mathrm{~g} \\
\text { Mango fresco }\end{array}$ & $\begin{array}{l}\text { Ingesta Diaria } \\
\text { Recomendada }\end{array}$ \\
\hline Mango fresco & $16,9+0,1^{\mathrm{a}}$ & $1,7+0,01$ & $14,9+0,1^{\mathrm{a}}$ & $2,0+0,2$ \\
\hline Mango fortificado & $375,5+2,8^{b}$ & $37,6+0,3$ & $351,3+2,7^{c}$ & $43,9+0,3$ \\
\hline
\end{tabular}


gativos) acentuados con la disolución de fortificación, condición que permitió alcanzar niveles mayores de $\mathrm{Ca}^{+2}$ en comparación con el criterio de fortificación fijado teóricamente (20\% IDR/200 g mango fresco). La fruta se expande durante el proceso de IV (valores positivos de deformación $\left(\gamma_{1}\right.$ y $\left.\gamma\right)$.

\section{RESUMEN}

La impregnación al vacío es una tecnología eficaz para la incorporación de compuestos fisiológicamente activos (CFA) en la matriz porosa de un alimento dentro del marco de los mínimamente procesados, proporcionando nuevas categorías de productos funcionales que responden a las necesidades de mejorar la calidad nutricional de la población con carencia en micronutrientes. Fueron utilizados cilindros de mango fresco impregnados a vacío con una solución de lactato de calcio $6 \%$ y cloruro de calcio $1 \%$. Las condiciones del proceso (presión y tiempo de vacío) y tamaño de la muestra fueron definidas a través del estudio cuyas interacciones afectaron significativamente los parámetros de impregnación $\mathrm{X}$ y $\varepsilon_{\mathrm{e}}$. La técnica permitió incorporar un 37,6\% de la Ingesta Diaria Recomendada (IDR) de calcio en $200 \mathrm{~g}$ de mango fresco, variedad Tommy Atkins, sin afectar las características sensoriales del producto final. La determinación de calcio fue realizada por espectrofotometría de absorción atómica. El proceso generó pérdidas de líquidos nativos, expansión de la fruta y disminución de los valores de $\mathrm{X}$ y $\varepsilon_{\mathrm{e}}$ por la presencia de iones calcio en el mango impregnado.

Palabras clave: Alimentos fortificados, alimentos funcionales, minerales, frutas, mango.

Dirigir la correspondencia a:

Profesora

Amanda Consuelo Díaz Moreno

Instituto de Ciencia y Tecnología de Alimentos

ICTA.

Universidad Nacional de Colombia, Colombia

Teléfono: 57(1)3165000 extensión 19032

E-mail: amcdiazmo@unal.edu.co

\section{BIBLIOGRAFÍA}

1. Fito P. Chiralt A. y Pardo P. Coupling of hydrodynamic mechanism and deformation-relaxation phenomena during vacuum teatements in solid porous food-liquid systems. J Food Engineering 1996; 27(3): 229-40.

2. Laurindo J. Stringari J. Paes S. y Carciofi B. Experimental Determination of the Dynamics of Vacuum Impregnation of Apples. J Food Sci 2007; 72(8): 470-5.
3. Fito P. Modelling of vacuum osmotic dehydration of foods. J Food Engineering 1994; 22 (4): 313 - 8.

4. Moraga M. Moraga G. Fito P. y Martínez-Navarrete N.Effect of vacuum impregnation with calcium lactate on the osmotic dehydration kinetics and quality of osmodehydrated grapefruit. J Food Engineering 2009; 90: 372-9.

5. Lin D. Leonard S. Lederer C. Traber M. y Zhao Y. Retention of fortified vitamin $\mathrm{E}$ and sensory quality of fresh-cut pears by vacuum impregnation with honey. J Food Sci 2006; 71(7): 553-9.

6. Park S. Kodihalli I. y Zhao Y. Nutritional, sensory, and physicochemical properties of vitamin E- and mineral-fortified fresh-cut apples by use of vacuum impregnation. J Food Sci 2005; 70(9): 593 - 9.

7. Hironaka K. Kikuchi M. Koaze H. Sato T. Kojima M. Yamamoto K. Yasuda K. Mori M. y Tsuda S. Ascorbic acid enrichment of whole potato tuber by vacuum-impregnation. Food Chem 2011; 127 : 1114-8.

8. Barrera C. Betoret N. y Fito P. Ca and Fe influence on the osmotic dehydration kinetics of apple slices (Granny Smith). J Food Engineering, 2004;65:9-14.

9. Perez-Cabrera L. Chafer M. Chiralt A. y GonzalezMartinez C. Effectiveness of antibrowning agents applied by vacuum impregnation on minimally processed pear, LWT - Food Sci Technol 2011; 44: 2273-80.

10. Guillemin A. Degraeve P. Guillon F. Lahaye M. y Saurel R. Incorporation of pectinmethylesterase in apple tissue either by soaking or by vacuumimpregnation. Enzyme Microbial Technol 2006; 38: 610-6.

11. Derossi A. De Pilli T. y Severini C. Reduction in the $\mathrm{pH}$ of vegetables by vacuum impregnation: A study on pepper. J Food Engineering 2010; 99: 9-15.

12. Menrad K. Market and marketing of functional food in Europe. J Food Engineering 2003; 56: 181-8.

13. Allende A. Tomás-Barberán F. y Gil M. Minimal processing for healthy traditional foods. Trends Food Sci Technol 2006; 17: 513 - 9.

14. Instituto Colombiano de Bienestar Familiar (ICBF). Encuesta Nacional de la Situación Nutricional en Colombia - ENSIN. 2005.

15. Del Valle J. M., Arhguiz V. y Díaz L. Volumetric Procedure to Assess Infiltration Kinetics and Porosity of Fruits by Applying a Vacuum Pulse. J Food Engineering 1998; 38, 207-21.

16. Cortés M. Guardiola L. y Pacheco R. Aplicación de la ingeniería de matrices en el enriquecimiento de mango (var. Tommy Atkins) con calcio. DYNA 2007; 74(1): 19-26. 
17. Muntada V. Gerschenson L. Alzamora S. y Castro M. Solute Infusion Effects on Texture of Minimally Processed Kiwifruit. J Food Sci 1998; 63(4): 616-20.

18. Lorenzo J. García M. Franco I. y Carballo J. Biochemical characteristics of dry-cured lacón (a Spanish traditional meat product) throughout the manufacture, and sensorial properties of the final product. Food Control 2008; 19: 1148-58.

19. Mujica-Paz H. Valdez-Fragoso A. Lopez-Malo A. y Palou E. Impregnation properties of some fruits at vacuum pressure. J Food Engineering 2003; 56: $307-14$.

20. Salvatori D. Chiralt A. y Fito P. The response of some properties of fruits to vacuum impregnation. J Food Process Engineering 1998; 21: 59 - 73.

21. Guillemin A. Degraeve P. Noël C. y Saurel R. Influence of impregnation solution viscosity and osmolarity on solute uptake during vacuum impregnation of apple cubes (var. Granny Smith). J Food Engineering 2008; 86: 475-83. 